\title{
The completion of the classification of the regular near octagons with thick quads
}

\author{
Bart De Bruyn*
}

Received: 25 February 2005 / Accepted: 16 November 2005

(C) Springer Science + Business Media, LLC 2006

\begin{abstract}
Brouwer and Wilbrink [3] showed the nonexistence of regular near octagons whose parameters $s, t_{2}, t_{3}$ and $t$ satisfy $s \geq 2, t_{2} \geq 2$ and $t_{3} \neq t_{2}\left(t_{2}+1\right)$. Later an arithmetical error was discovered in the proof. Because of this error, the existence problem was still open for the near octagons corresponding with certain values of $s$, $t_{2}$ and $t_{3}$. In the present paper, we will also show the nonexistence of these remaining regular near octagons.
\end{abstract}

MSC2000 05B25, 05E30, 51E12

Keywords (regular) near polygon

\section{Introduction}

A near polygon ([9]) is a partial linear space $\mathcal{S}=(\mathcal{P}, \mathcal{L}, \mathrm{I}), \mathrm{I} \subseteq \mathcal{P} \times \mathcal{L}$, with the property that for every point $x \in \mathcal{P}$ and every line $L \in \mathcal{L}$, there exists a unique point on $L$ nearest to $x$. Here distances are measured in the point graph or collinearity graph of $\mathcal{S}$. If $d$ is the diameter of $\mathcal{S}$, then the near polygon is called a near $2 d$-gon. The unique near 0 -gon consists of one point (no lines). The near 2-gons are precisely the lines. Near quadrangles are usually called generalized quadrangles (GQ's, [7]). We call a generalized quadrangle thick if every line is incident with at least three points and if every point is incident with at least three lines.

If $A$ and $B$ are two nonempty sets of points, then $\mathrm{d}(A, B)$ denotes the minimal distance between a point of $A$ and a point of $B$. If $A$ is a singleton $\{x\}$, then we will

*Postdoctoral Fellow of the Research Foundation - Flanders

B. D. Bruyn $(\square)$

Department of Pure Mathematics and Computer Algebra, Ghent University, Galglaan 2, B-9000 Gent, Belgium

E-mail: bdb@cage.ugent.be 
also write $\mathrm{d}(x, B)$ instead of $\mathrm{d}(\{x\}, B)$. If $A$ is a nonempty set of points and if $i \in \mathbb{N}$, then we denote by $\Gamma_{i}(A)$ the set of points $y$ for which $\mathrm{d}(y, A)=i$. If $A$ is a singleton $\{x\}$, then we also write $\Gamma_{i}(x)$ instead of $\Gamma_{i}(\{x\})$.

A near $2 n$-gon, $n \geq 1$, is said to have $\operatorname{order}(s, t)$ if every line is incident with precisely $s+1$ points and if every point is incident with precisely $t+1$ lines. A near $2 n$-gon, $n \geq 1$, is called regular if its point graph is a so-called distance-regular graph ([2]), or equivalently, if it has an order $(s, t)$ and if there exists constants $t_{i}$, $i \in\{0, \ldots, n\}$, such that for any two points $x$ and $y$ at distance $i$ from each other, there are precisely $t_{i}+1$ lines through $y$ containing a point at distance $i-1$ from $x$. Obviously, $t_{0}=-1, t_{1}=0$ and $t_{n}=t$.

A sub near polygon $\mathcal{S}^{\prime}$ of a near polygon $\mathcal{S}$ is called geodetically closed if it satisfies the following properties:

(i) the points of $\mathcal{S}^{\prime}$ determine a subspace of $\mathcal{S}$;

(ii) every point of on a shortest path (in $\mathcal{S}$ ) between two points of $\mathcal{S}^{\prime}$ is again a point of $\mathcal{S}^{\prime}$.

A near polygon is called dense if every line is incident with at least three points and if every two points at distance 2 have at least two common neighbours. By [3, Theorem 4], every two points of a dense near polygon at distance $\delta$ from each other are contained in a unique geodetically closed sub near $2 \delta$-gon. These sub near polygons are called quads if $\delta=2$ and hexes if $\delta=3$.

In [3, Theorem 7], Brouwer and Wilbrink showed the nonexistence of regular near octagons whose parameters $s, t_{2}, t_{3}$ and $t$ satisfy the following conditions: $s \geq 2, t_{2} \geq 2$ and $t_{3} \neq t_{2}\left(t_{2}+1\right)$. Later an arithmetical error was discovered in the proof (lines 5 and 6 of page 172), causing a gap in the proof. The authors of [2] ask in their book to fill this gap (see page 206) and in the present paper we will do that.

The regular near octagons which still need to be ruled out all have parameters of the following form: $t_{2}=q, s=q^{2}$ and $q^{4}+q^{3}+2 q \leq t_{3} \leq q^{4}+q^{3}+q^{2}+2 q+18$. The lower bound for $t_{3}$ arises from the divisibility condition $t_{2} \mid t_{3}$ and the inequality $t_{3}+1>\left(t_{2}+1\right)\left(s t_{2}+1\right)$ which are know to hold for any nonclassical dense regular near hexagon which is not isomorphic to the $M_{24}$ near hexagon. We will rule out the remaining regular near octagons by improving this lower bound for $t_{3}+1$ and subsequently dealing with the remaining cases. As a consequence, we have

Theorem 1 ([3, Theorem 7] + Section 4). There exist no regular near octagons whose parameters $s, t_{2}, t_{3}$ and $t$ satisfy $s \geq 2, t_{2} \geq 2$ and $t_{3} \neq t_{2}\left(t_{2}+1\right)$.

\section{Some properties of generalized quadrangles}

As we mentioned before the generalized quadrangles are precisely the near quadrangles. Any generalized quadrangle which is not degenerate, not a grid and not a dual grid must have a certain order $\left(s, t_{2}\right)$. The aim of this section is to collect some known and easy properties of generalized quadrangles.

Lemma 1 ([7, 1.2.2 and 1.2.3]). If $Q$ is a generalized quadrangle of order $\left(s, t_{2}\right)$, then 
- $s+t_{2} \mid s t_{2}(s+1)\left(t_{2}+1\right)$;

- $s \leq t_{2}^{2}$ if $t_{2} \neq 1$, or dually, $t_{2} \leq s^{2}$ if $s \neq 1$ (Higman's inequality).

Lemma 2 ([7, 2.2.1]). If $Q$ is a generalized quadrangle of order $\left(s, t_{2}\right)$ and if $Q^{\prime}$ is a proper subquadrangle of order $\left(s, t_{2}^{\prime}\right)$ of $Q$, then $t_{2}^{\prime} \leq \frac{t_{2}}{s}$. Equality holds if and only if every line of $Q$ meets $Q^{\prime}$.

Lemma 3 ([7, 2.3.1]). Let $Q$ be a generalized quadrangle of order $\left(s, t_{2}\right), s \neq 1$, and let $X$ be a nonempty set of points of $Q$. If every line of $Q$ which has at least two points in common with $X$ is completely contained in $X$, then $X$ is one of the following sets:

(a) a set of mutually noncollinear points;

(b) the set of points on a pencil of lines (i.e. a set of lines through a distinguished point);

(c) the set of points of a subquadrangle of order $\left(s, t_{2}^{\prime}\right)$.

\section{Restrictions on the parameters of regular near hexagons}

Let $\mathcal{S}$ be a regular near hexagon with parameters $s, t_{2}$ and $t_{3}$ and let $A$ denote the collinearity matrix of $\mathcal{S}$. There exist well-known techniques for calculating the eigenvalues and corresponding multiplicities of $A$, see e.g. [2] or Section 7 of [8]. The graph $A$ has four distinct eigenvalues $\lambda_{i}, i \in\{0,1,2,3\}$, with $-\left(t_{3}+1\right)=\lambda_{0}<\lambda_{1}<\lambda_{2}<$ $\lambda_{3}=s\left(t_{3}+1\right)$. Here $\lambda_{1}$ and $\lambda_{2}$ are the roots of the quadratic polynomial

$$
X^{2}-(s-1)\left(t_{2}+2\right) X+\left(s^{2}-s+1\right) t_{2}-s t_{3}+(s-1)^{2} .
$$

The multiplicity $f_{3}$ of the eigenvalue $s\left(t_{3}+1\right)$ is equal to 1 . The multiplicity $f_{0}$ of the eigenvalue $-\left(t_{3}+1\right)$ is equal to

$$
s^{3} \frac{\left(t_{2}+1\right)+s t_{3}\left(t_{2}+1\right)+s^{2} t_{3}\left(t_{3}-t_{2}\right)}{s^{2}\left(t_{2}+1\right)+s t_{3}\left(t_{2}+1\right)+t_{3}\left(t_{3}-t_{2}\right)},
$$

and the multiplicity $f_{3-i}$ of the eigenvalue $\lambda_{3-i}, i \in\{1,2\}$, is equal to

$$
\frac{\lambda_{i}(m-1)+s\left(t_{3}+1\right)-\left(\lambda_{i}+t_{3}+1\right) f_{0}}{\lambda_{i}-\lambda_{3-i}},
$$

where

$$
m=1+s\left(t_{3}+1\right)+\frac{s^{2}\left(t_{3}+1\right) t_{3}}{t_{2}+1}+\frac{s^{3} t_{3}\left(t_{3}-t_{2}\right)}{t_{2}+1} .
$$

The fact that all these multiplicities are integers gives already severe restrictions on the parameters. Another restriction is the so-called Mathon bound ([3, 5, 6]) which holds if $s \neq 1$ :

$$
t_{3} \leq s^{3}+t_{2}\left(s^{2}-s+1\right)
$$


Another inequality which holds if $s$ is different from 1 is the following:

$$
t_{3}^{2}-\left(s^{2} t_{2}+s^{2}+t_{2}\right) t_{3}+s^{4}\left(t_{2}+1\right) \geq 0
$$

This inequality follows from one of the Krein conditions, see Section (i) of [3] or Remark 2.4 of [6].

Suppose now that $\mathcal{S}$ is dense, so suppose that $s \geq 2$ and $t_{2} \geq 1$. Then every two points at distance 2 are contained in a unique quad of order $\left(s, t_{2}\right)$. So, Lemma 1 provides additional parameter restrictions. The number of quads through a point, respectively line, is equal to $\frac{t_{3}\left(t_{3}+1\right)}{t_{2}\left(t_{2}+1\right)}$, respectively $\frac{t_{3}}{t_{2}}$. Hence, we also obtain the following divisibility conditions:

$$
\begin{gathered}
t_{2} \mid t_{3} ; \\
t_{2}\left(t_{2}+1\right) \mid t_{3}\left(t_{3}+1\right) .
\end{gathered}
$$

Now, let $Q$ denote an arbitrary quad of $\mathcal{S}$. If $x$ is a point of $\Gamma_{1}(Q)$, then a unique line through $x$ meets $Q$ and $t_{2}\left(t_{2}+1\right)$ lines through $x$ are completely contained in $\Gamma_{1}(Q)$. As a consequence, $t_{3} \geq t_{2}\left(t_{2}+1\right)$. Moreover, $t_{3}=t_{2}\left(t_{2}+1\right)$ if and only if $\Gamma_{2}(R)=\emptyset$ for every quad $R$, or equivalently, if and only if $\mathcal{S}$ is a so-called classical near hexagon (i.e. a dual polar space of rank 3 ([4])). If $t_{3} \neq t_{2}\left(t_{2}+1\right)$, then there exists a point $x \in \Gamma_{2}(Q)$. The set $\Gamma_{2}(x) \cap Q$ is an ovoid of $Q$ and the set of $s t_{2}+1$ quads through $x$ which meet $Q$ determine $\left(t_{2}+1\right)\left(s t_{2}+1\right)$ distinct lines through $x$ (see Lemma 25 of [3]). So,

$$
\left(t_{3}+1\right) \geq\left(t_{2}+1\right)\left(s t_{2}+1\right) .
$$

If $t_{3}+1=\left(t_{2}+1\right)\left(s t_{2}+1\right)$, then Theorem 5 of [3] shows that $s=2, t_{2}=2$ and $t=14$. By [1], there is a unique near hexagon with these parameters, namely the near hexagon which is obtained in the following way from the unique Steiner system $S(5,8,24)$ (= the threefold extension of $\mathrm{PG}(2,4))$ :

- the points of the near hexagon are the blocks of $S(5,8,24)$;

- the lines are the triples of mutually disjoint blocks;

- incidence is containment.

We will refer to this near hexagon as the $M_{24}$ near hexagon since its automorphism group is isomorphic to the Mathieu group $M_{24}$.

Theorem 2. Let $\mathcal{S}$ be a nonclassical regular near hexagon with parameters $s \geq 2$, $t_{2} \geq 1$ and $t_{3}$ which is not isomorphic to the $M_{24}$ near hexagon. Let $t_{2}^{\prime} \in \mathbb{N}$ such that no quad of $\mathcal{S}$ has subquadrangles of order $(s, \alpha)$ with $t_{2}^{\prime}<\alpha \leq \frac{t_{2}-1}{s}$. Then $t_{3}+1=$ $\left(s t_{2}+1\right)\left(t_{2}+1\right)+\eta \cdot t_{2}$ with $\eta \geq \min \left\{t_{2}, \frac{\left(t_{2}+1\right)\left(s t_{2}+1\right)}{s t_{2}^{\prime}+1}-\left(s t_{2}+s+1\right)\right\}$.

Proof: Since $t_{3}+1 \geq\left(t_{2}+1\right)\left(s t_{2}+1\right)$ and $t_{2} \mid t_{3}$, there exists an $\eta \in \mathbb{N}$ such that $t_{3}+1=\left(s t_{2}+1\right)\left(t_{2}+1\right)+\eta \cdot t_{2}$. Let $R$ denote an arbitrary quad of $\mathcal{S}$. Since $\mathcal{S}$ is not classical, $t_{3} \neq t_{2}\left(t_{2}+1\right)$ and there exists a point $x \in \Gamma_{2}(R)$. Since $\mathcal{S}$ is not isomorphic to the $M_{24}$ near hexagon, $t_{3}+1>\left(t_{2}+1\right)\left(s t_{2}+1\right)$ and hence there exists a line $L$ 
through $x$ which is completely contained in $\Gamma_{2}(R)$. Let $V$ denote the set of lines of $\mathcal{S}$ which meet $L$ and $\Gamma_{1}(R)$. For every quad $Q$ through $L$, let $V_{Q}$ denote the set of lines of $V$ which are contained in $Q$. Since $|V|=(s+1)\left(s t_{2}+1\right)\left(t_{2}+1\right)$ and since there are $\frac{t_{3}}{t_{2}}$ quads through $L$, there exists a quad $Q^{*}$ through $L$ for which $\left|V_{Q^{*}}\right| \geq \frac{(s+1) t_{2}\left(s t_{2}+1\right)\left(t_{2}+1\right)^{2}}{t_{3}}$.

Put $X:=R \cup \Gamma_{1}(R)$. By Lemma 8 of [3], every line which has at least two points in common with $X$ is completely contained in $X$. Hence, if $Q$ is a quad through $L$, then either $Q \cap X$ is empty or satisfies the conditions of Lemma 3. We distinguish the following possibilities:

(i) $Q \cap X$ is a (possibly empty) set of mutually noncollinear points. In this case, $\left|V_{Q}\right|=|Q \cap X| \leq s t_{2}$.

(ii) $Q \cap X$ is the set of points on $k \geq 1$ lines through a distinguished point. Then $\left|V_{Q}\right|=1+s k \leq 1+s t_{2}$.

(iii) $Q \cap X$ determines a subquadrangle of $\operatorname{order}(s, \alpha)$. Since $Q$ contains a line which is disjoint from the subquadrangle, $\alpha \leq \frac{t_{2}-1}{s}$ by Lemma 2 . By our assumptions, $\alpha \leq t_{2}^{\prime}$. Hence, $\left|V_{Q}\right|=(s+1)(s \alpha+1) \leq(s+1)\left(s t_{2}^{\prime}+1\right)$.

Taking $Q=Q^{*}$, we see that $\frac{(s+1) t_{2}\left(s t_{2}+1\right)\left(t_{2}+1\right)}{t_{3}} \leq s t_{2}+1$ or $\frac{(s+1) t_{2}\left(s t_{2}+1\right)\left(t_{2}+1\right)}{t_{3}} \leq(s+$ $1)\left(s t_{2}^{\prime}+1\right)$. The first inequality is equivalent with $\eta \geq t_{2}$ and the latter with $\eta \geq$ $\frac{\left(t_{2}+1\right)\left(s t_{2}+1\right)}{s t_{2}^{\prime}+1}-\left(s t_{2}+s+1\right)$. The theorem now immediately follows.

Corollary 1. Let $\mathcal{S}$ be a nonclassical regular near hexagon with parameters $s \geq 2$, $t_{2} \geq 1$ and $t_{3}$ which is not isomorphic to the $M_{24}$ near hexagon. Suppose no quad of $\mathcal{S}$ has subquadrangles of order $(s, \alpha)$ with $\alpha \leq \frac{t_{2}-1}{s}$. Then $t_{3} \geq(s+1) t_{2}\left(t_{2}+1\right)$. In particular, this inequality holds if $t_{2} \leq s$.

Proof: By the proof of Theorem $2, \eta \geq t_{2}$ and hence $t_{3} \geq(s+1) t_{2}\left(t_{2}+1\right)$.

Remark. In Theorem 2 we can take for $t_{2}^{\prime}$ the biggest integer smaller than or equal to $\frac{t_{2}-1}{s}$ for which the divisibility condition $s+t_{2}^{\prime} \mid s(s+1) t_{2}^{\prime}\left(t_{2}^{\prime}+1\right)$ is satisfied. If $t_{2}^{\prime} \neq \frac{t_{2}-1}{s}$ (which is certainly the case if $\frac{t_{2}-1}{s} \notin \mathbb{N}$ ), then we get an improvement of the lower bound $\left(t_{2}+1\right)\left(s t_{2}+1\right)$ for $t_{3}$.

\section{The nonexistence of the regular near octagons}

\subsection{Description of the gap}

Suppose that $\mathcal{S}$ is a regular near octagon with parameters $s, t_{2}, t_{3}$ and $t$, with $s \geq 2$, $t_{2} \geq 2$ and $t_{3} \neq t_{2}\left(t_{2}+1\right)$. The case $s \neq t_{2}^{2}$ has been ruled out in [3]. So, suppose that there exists a $q \geq 2$ such that $t_{2}=q$ and $s=q^{2}$. For $q=2$, we have $s=4$, $t_{2}=2, t_{3} \geq\left(t_{2}+1\right)\left(s t_{2}+1\right)=27$ and $t_{3} \leq 90$ by Mathon's bound. Each value of $t_{3} \in\{27, \ldots, 90\}$ violates however at least one of the following conditions: (i) $t_{2} \mid t_{3}$, (ii) $t_{2}\left(t_{2}+1\right) \mid t_{3}\left(t_{3}+1\right)$, (iii) all multiplicities $f_{i}$ are integral. So, we may suppose that $q \geq 3$. Put

$$
f_{q}(x):=x^{2}-\left(q^{5}+q^{4}+q\right) x+\left(q^{9}+q^{8}\right) .
$$


For $q \geq 3$, this polynomial has two roots $r_{1}(q)$ and $r_{2}(q)$ with $0<r_{1}(q)<r_{2}(q)$. By Section 3 , we have $f_{q}\left(t_{3}\right) \geq 0$ and hence either $t_{3} \leq r_{1}(q)$ or $t_{3} \geq r_{2}(q)$. The case $t_{3} \geq$ $r_{2}(q)$ has been ruled out in [3]. The case $t_{3} \leq r_{1}(q)$ has not yet been ruled out because of an arithmetical error. Since $f_{q}\left(q^{4}+q^{3}+q^{2}+2 q+19\right)=-16 q^{5}+23 q^{4}+41 q^{3}+$ $40 q^{2}+57 q+361<0$ for every $q \geq 3, t_{3} \leq q^{4}+q^{3}+q^{2}+2 q+18$. Because $t_{3}>$ $\left(t_{2}+1\right)\left(s t_{2}+1\right)$ and since $t_{2}$ is a divisor of $t_{3}, q^{4}+q^{3}+2 q \leq t_{3}$. As a consequence,

$$
q^{4}+q^{3}+2 q \leq t_{3} \leq q^{4}+q^{3}+q^{2}+2 q+18 .
$$

This is precisely the description of the gap as given on page 206 of [2].

\subsection{Filling of the gap}

By Corollary 1 , we have $t_{3} \geq(s+1) t_{2}\left(t_{2}+1\right) \geq q^{4}+q^{3}+q^{2}+q$. Since $q \mid t_{3}$ and $t_{3} \leq q^{4}+q^{3}+q^{2}+2 q+18$, either $t_{3}=q^{4}+q^{3}+q^{2}+q, t_{3}=q^{4}+q^{3}+q^{2}+$ $2 q$ or $q^{4}+q^{3}+q^{2}+3 q \leq t_{3} \leq q^{4}+q^{3}+q^{2}+2 q+18$. (So, $q \leq 18$ in the latter case.) We will kill each of these cases in the following lemmas.

Lemma 4. The case $t_{3}=q^{4}+q^{3}+q^{2}+q$ cannot occur.

Proof: In this case the multiplicity of the eigenvalue $-\left(t_{3}+1\right)$ is equal to

$$
\begin{aligned}
& \frac{q^{15}+2 q^{14}+3 q^{13}+3 q^{12}+2 q^{11}+2 q^{10}+2 q^{9}+2 q^{8}+2 q^{7}+q^{6}+q^{4}+q^{3}}{q^{5}+3 q^{4}+5 q^{3}+6 q^{2}+5 q+2} \\
& =q^{10}-q^{9}+q^{8}-q^{7}+q^{6}+q^{5}-3 q^{4}+3 q^{3}-q^{2}-\frac{2 q^{4}-2 q^{2}}{q^{5}+3 q^{4}+5 q^{3}+6 q^{2}+5 q+2} .
\end{aligned}
$$

Since $0<2 q^{4}-2 q^{2}<q^{5}+3 q^{4}+5 q^{3}+6 q^{2}+5 q+2$, this multiplicity would not be integral, a contradiction.

Lemma 5. The case $t_{3}=q^{4}+q^{3}+q^{2}+2 q$ cannot occur.

Proof: In this case the multiplicity of the eigenvalue $-\left(t_{3}+1\right)$ is equal to

$$
\frac{q^{16}+2 q^{15}+3 q^{14}+5 q^{13}+4 q^{12}+4 q^{11}+4 q^{10}+2 q^{9}+3 q^{8}+2 q^{7}+q^{5}+q^{4}}{q^{6}+3 q^{5}+5 q^{4}+8 q^{3}+8 q^{2}+5 q+2}
$$

or to

$$
\begin{aligned}
& q^{10}-q^{9}+q^{8}-q^{7}+2 q^{6}-2 q^{5}+3 q^{4}-8 q^{3}+15 q^{2}-19 q+28 \\
& -\frac{55 q^{5}+73 q^{4}+131 q^{3}+159 q^{2}+102 q+56}{q^{6}+3 q^{5}+5 q^{4}+8 q^{3}+8 q^{2}+5 q+2} .
\end{aligned}
$$

If $q \geq 54$, then $0<55 q^{5}+73 q^{4}+131 q^{3}+159 q^{2}+102 q+56<q^{6}+3 q^{5}+$ $5 q^{4}+8 q^{3}+8 q^{2}+5 q+2$, contradicting the fact that the multiplicity is integral. So, $3 \leq q \leq 54$. Also for the remaining possibilities of $q$ one can verify (individually) that Springer 
the multiplicity of the eigenvalue $-\left(t_{3}+1\right)$ is not integral. So, also this case cannot occur.

Lemma 6. The case $q^{4}+q^{3}+q^{2}+3 q \leq t_{3} \leq q^{4}+q^{3}+q^{2}+2 q+18$ cannot occur.

Proof: If $q \geq 6$, then $t_{3} \geq q^{4}+q^{3}+q^{2}+2 q+6$. On the other hand, since $f_{q}\left(q^{4}+\right.$ $\left.q^{3}+q^{2}+2 q+6\right)=-3 q^{5}+10 q^{4}+15 q^{3}+14 q^{2}+18 q+36<0$ if $q \geq 6, t_{3}<$ $q^{4}+q^{3}+q^{2}+2 q+6$. So, we have a contradiction.

If $q=5$, then $q^{4}+q^{3}+q^{2}+3 q \leq t_{3} \leq r_{1}(q)$ implies that $t_{3}=790$. But for this value of $t_{3}$, the divisibility condition $t_{2}\left(t_{2}+1\right) \mid t_{3}\left(t_{3}+1\right)$ is not satisfied.

If $q=4$, then $q^{4}+q^{3}+q^{2}+3 q \leq t_{3} \leq r_{1}(q)$ implies that $348 \leq t_{3} \leq 351$. No possible value of $t_{3}$ survives the conditions $t_{2} \mid t_{3}$ and $t_{2}\left(t_{2}+1\right) \mid t_{3}\left(t_{3}+1\right)$.

If $q=3$ then $q^{4}+q^{3}+q^{2}+3 q \leq t_{3} \leq r_{1}(q)$ implies that $126 \leq t_{3} \leq 141$. From $t_{2} \mid t_{3}$ and $t_{2}\left(t_{2}+1\right) \mid t_{3}\left(t_{3}+1\right), t_{3} \in\{132,135\}$. None of the possible values of $t_{3}$ gives rise a integral multiplicity for the eigenvalue $-\left(t_{3}+1\right)$.

Acknowledgements The author wants to thank both referees for their suggested improvements regarding the proof of Theorem 2 .

\section{References}

1. A. E. Brouwer, "The uniqueness of the near hexagon on 759 points," in N. L. Johnson, M. J. Kallahar, and C. T. Long, (eds.), Finite Geometries, volume 82 of Lecture Notes in Pure and Appl. Math., Marcel Dekker, New York, Basel, 1982, pp. 47-60.

2. A. E. Brouwer, A. M. Cohen, and A. Neumaier, Distance-Regular Graphs. Springer-Verlag, Berlin, 1989.

3. A. E. Brouwer and H. A. Wilbrink, "The structure of near polygons with quads," Geom. Ded., 14 (1983), $145-176$.

4. P. J. Cameron, "Dual polar spaces,” Geom. Dedicata, 12 (1982), 75-86.

5. R. Mathon, "On primitive association schemes with three classes," preprint.

6. A. Neumaier, "Krein conditions and regular near polygons," J. Combin. Theory Ser. A, 54 (1990), 201209.

7. S. E. Payne and J. A. Thas, Finite Generalized Quadrangles, volume 110 of Research Notes in Mathematics. Pitman, Boston, 1984.

8. S. Shad and E. E. Shult, "The near $n$-gon geometries," preprint.

9. E. E. Shult and A. Yanushka, "Near n-gons and line systems," Geom. Dedicata, 9 (1980), 1-72. 